\title{
ENERGY RATIO OF REFLECTED AND REFRACTED SEISMIC WAVES*
}

\author{
By B. Gutenberg
}

\section{\$1. INTRODUCTION}

THE DISPLACEMENT $D$ of a point at the surface of the earth produced by body waves originating at a distant source of elastic disturbance is given by

$$
D=C T Q \sqrt{\left(F_{1} F_{2} \cdots F_{n}\right) a \frac{d \cos i_{h} / d \Delta}{\sin \Delta \cos i_{0}}}
$$

where $C$ depends on the energy radiating from the source, $T=$ period of arriving waves, $Q=$ ratio of ground displacement to incident amplitude, $F=$ ratio of transmitted or reflected energy to incident energy at each point where the wave has encountered a discontinuity in density or wave velocity or both, $a$ gives the effect of absorption, $i_{h}$ and $i_{0}$ are the angles of incidence of the wave at the source (depth $h$ ) and the point of arrival at the surface of the earth, respectively, and $\Delta$ is the angular distance between the source and the point of observation. $i_{0}, \Delta$ and $d \Delta / d i_{h}$ depend only on the wave velocity along the path and on $i_{h} ; Q$ is a function only of $i_{0}$ and of Poisson's ratio just below the surface of. the earth; the value of $F$ at a given discontinuity depends on the angle of incidence there, the densities and the wave velocities on both sides of the discontinuity, and the type of wave-longitudinal $(P)$ or transverse (SV in the plane of propagation or SH with vibrations perpendicular to the plane of propagation only). Thus, $Q$ and $F$ may be tabulated as a function of the angle of incidence $i$ for any given discontinuity and type of waves.

The purpose of the present paper is the investigation of $Q$ corresponding to various values of Poisson's ratio, and of $F$ under assumptions which represent conditions likely to occur at discontinuities between the crustal layers of the earth. Some of the results may also be useful for calculations of amplitudes in seismic prospecting.

\section{$\S$ II. THEORY}

$\S$ II a) General equations. - The first equations which permitted the calculation of the energy ratios $F$ were given by Knott (1899). The factors $A_{1} / A, A^{\prime} / A$, etc., used by Knott may be represented by the letter $R .{ }^{1}$ Then, the relationship between $R$ and $F$ is given by

$$
F=R^{2} \frac{\rho_{2}}{\rho_{1}} \frac{\cot i_{2}}{\cot i_{1}}
$$

where $\rho=$ density, $i=$ angle of incidence, subscript 1 refers to the incident wave, and subscript 2 to the reflected or refracted wave as the case may be. For the reflected waves, $\rho_{2}=\rho_{1}$.

Equations for the calculation of the amplitude ratios $A$ of the reflected or refracted to the incident waves were developed by Zoeppritz in 1907 and published

* Manuscript received for publication June 30, 1943.

1 Knott's tabulated and plotted results refer to $F$ and not to $R$. 
eleven years after his death (Zoeppritz, 1919). The relationship between $A$ and $F$ is given by

$$
F=A^{2} \frac{\rho_{2}}{\rho_{1}} \frac{\sin 2 i_{2}}{\sin 2 i_{1}}
$$

Both sets of equations, those of Knott and those of Zoeppritz as well, have been used and reproduced repeatedly. Unfortunately, misprints are not infrequent. ${ }^{2}$ Further contributions to the theory have been published; for example, by Jeffreys (1926), Blut (1932), Muskat (1938), Dix (1939), Joos and Teltow (1939), Muskat and Meres (1940), Ott (1942). An excellent summary of the fundamental papers, with a discussion of the relations between some of the equations and quantities used, was given by Macelwane (1936, pp. 147-179). For certain purposes, the use of the Zoeppritz equations is preferable; some consider the Knott equations as less tedious to work with. The author, in special instances, has used with advantage equations giving directly the square roots of the energy ratios $F$ of the reflected or refracted to the incident energy, which are to be used in the evaluation of amplitudes from equation (1). At first glance, these equations appear to be more complicated than those of Knott or Zoeppritz, but it must be considered that they give directly the desired quantities, while to each value resulting from the equations of Knott or Zoeppritz equations (2) and (3), respectively, must be applied to find the quantities needed.

Throughout the present paper the following symbols are used:

$\mathrm{P}=$ longitudinal wave

$\mathrm{SV}=$ component of $\mathrm{S}$ in the plane of propagation

Square root of energy ratio $F$ Angle of incidence Velocity

Density in medium Rigidity in medium Poisson's ratio in medium $j=\sqrt{\rho_{1} / \rho_{2}}$

$n=\frac{V_{2}}{V_{1}} \quad q=\frac{V_{1}}{v_{1}} \quad p=\frac{v_{2}}{v_{1}} \quad r=\frac{v_{2}}{V_{1}}=\frac{p}{q}$
$\mathrm{S}=$ transverse wave

$\mathrm{SH}=$ component perpendicular to it. Transmitted

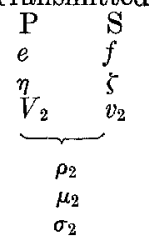

The following are fundamental equations:

$$
\begin{gathered}
\sigma_{1}=\frac{1}{2}\left(1-\frac{1}{q^{2}-1}\right), \quad \text { similarly } \sigma_{2} \\
\sin a: \sin \beta: \sin \eta: \sin \xi=V_{1}: v_{1}: V_{2}: v_{2} \\
c^{2}+d^{2}+e^{2}+f^{2}=1
\end{gathered}
$$

If a $\mathrm{P}$ wave is incident, the square roots of the energy ratios are given by

$$
1+c+I d-j J e+j K f=0
$$

${ }_{2}^{2}$ For example, in Gutenberg (1932), p. 45, the last term in the third and fourth equations of the group (115) should contain the angle $2 i_{2}$ in the last terms (not $i_{2}$ ); on $p .52$, the last term of the last equation in (142) should contain the factor $V_{2} / \mathfrak{P}_{1}$ (not $\mathfrak{B}_{2} / \mathfrak{P}_{1}$ ); in Macelwane (1936), p. 169, equation $(7.66)$, the same misprint occurs in the term with $E$ (center of the line). However, in Macelwane (1933), p. 117, the corresponding equation in (5) is correct. In Byerly (1942), p. 167, equation $(62)$, one factor, $\rho_{2} / \rho_{1}$, should be omitted in the last term. 
ENERGY RATIO OF REFLECTED AND REFRACTED SEISMIC WAVES

$$
\begin{array}{r}
1-c+\frac{1}{I} d-\frac{j}{J} e-\frac{j}{K} f=0 \\
-1+c+L d+\frac{p^{2}}{j} M e-\frac{t}{j} Q f=0 \\
-1-c+\frac{1}{L} d+\frac{1}{j} J N e+\frac{r}{j} R f=0
\end{array}
$$

Similarly for an incident SV-wave

$$
\begin{array}{r}
-1+d-I c-j \frac{I}{K} f-j \frac{I}{J} e=0 \\
1+d+\frac{1}{I} c+j \frac{K}{I} f-j \frac{J}{I} e=0 \\
1+d-L c+\frac{p^{2}}{j} \frac{K}{I} f+\frac{s}{j} U e=0 \\
-1+d+\frac{1}{L} c-\frac{1}{j} \frac{I}{K} N f+\frac{p^{2}}{j s} \frac{N}{U} e=0
\end{array}
$$

In both groups of equations

$$
\begin{aligned}
& I=\sqrt{\cot a \cot \beta} ; J=\sqrt{\cot a \tan \eta} ; K=\sqrt{\cot a \cot \zeta} ; \frac{I}{K}=\sqrt{\cot \beta \tan \zeta} \\
& L=q \sqrt{\frac{\cot 2 \beta \cos 2 \beta}{\sin 2 \alpha}}=\frac{\cot ^{2} \beta-1}{2 I} ; \quad M=\sqrt{\frac{\cos \eta}{\cos \alpha}} ; \quad N=\frac{\cos 2 \zeta}{\cos 2 \beta} ; \\
& Q=\sqrt{\frac{\cot 2 \zeta \cos 2 \zeta}{\sin 2 a}} ; \quad R=\frac{\sqrt{\sin 2 \zeta \sin 2 \alpha}}{\cos 2 \beta} ; \quad U=\frac{\cos 2 \zeta}{\sqrt{\sin 2 \beta \sin 2 \eta}}
\end{aligned}
$$

If an SH wave is incident, there is only a reflected and possibly a transmitted $\mathrm{SH}$ wave. The square roots of their energy ratios are given by

where

$$
d=\frac{1-H}{1+H} \quad f=\frac{2}{1+H} \sqrt{I}
$$

$$
H=\frac{\rho_{2} v_{2} \cos \zeta}{\rho_{1} v_{1} \cos \beta}=\frac{\rho_{2} \sin 2 \zeta}{\rho_{1} \sin 2 \beta}=\frac{\mu_{2} \cot \zeta}{\mu_{1} \cot \beta}
$$

In the preceding equations, the plus sign in connection with a longitudinal wave corresponds to a movement in the direction from the medium of the incident wave to the medium of the transmitted wave. In the refracted $\mathrm{P}$ waves, the plus sign indicates that an arriving compression is transmitted as a compression or that a dilatation continues as a dilatation; a change in sign corresponds to a change from compression to dilatation and vice versa. In the reflected $\mathrm{P}$ waves, the minus sign indicates no change, the plus sign that the reflected wave starts with a dilatation if the arriving wave begins with a compression, and vice versa. In the transverse waves 
no generally adopted rule exists. Different usage of the plus and minus signs may be found even in two papers by the same author. Of course, the same rule should be used throughout. A change in sign with increasing angle of incidence usually indicates that the amplitude passes through zero.

All equations for the calculation of energy or amplitudes mentioned thus far are based on certain simplifications, especially the assumption of plane waves. In general, the results of the calculations are in good agreement with the observations. However, for a wave which travels almost tangent to the discontinuity (angle of incidence near $90^{\circ}$ ) and in the medium with the higher velocity, the calculated energy may be much too small. (Joos and Teltow, 1939, Ott, 1942; see also Muskat, 1938, Dix, 1939.)

Results for special cases may be found easily from the equations. For example, for:

1) $\alpha=0, \beta=0$.

$$
\text { P wave incident } \quad d=0, \quad f=0, \quad c=\frac{n-j^{2}}{n+j^{2}} \quad e=\frac{2 j \sqrt{n}}{n+j^{2}}
$$

$\left.\begin{array}{l}\text { SV wave incident } \\ \text { SH wave incident }\end{array}\right\} \quad c=0, \quad e=0, \quad d=-\frac{p-j^{2}}{p+j^{2}} \quad f=-\frac{2 j \sqrt{p}}{p+j^{2}}$

2) $a=90^{\circ}$

$$
\mathrm{P} \text { wave incident } \quad d=0, \quad e=0, \quad f=0, \quad c=-1
$$

SV wave incident $\quad c=0$, for others use (6).

3) $\beta=90^{\circ}$

$$
\text { SV or SH wave incident } c=0, \quad e=0, \quad f=0, \quad|d|=1
$$

In general, if the angle of incidence of the arriving wave is $90^{\circ}$, all the energy goes into the reflected wave of the same type; if any other angle of incidence is $90^{\circ}$, the calculated energy of the corresponding wave is zero.

\$ II b) Equations assuming equal velocities on both sides of discontinuity.-Usually the density as well as the elastic constants of a crystalline material are greater than those of the same material at a temperature above its melting point. Although experimental data for the rocks at the depth in the interior of the earth where this melting point is reached are completely lacking, we must consider the possibility that the ratio of the two densities is about equal to that of the elastic constants and that the change in wave velocity between the two states is small as first pointed out by Daly. For this reason the special equations are of interest which result from equations (5-11), if $n=p=1, a=\eta, \beta=\zeta$.

If a $\mathrm{P}$ wave is incident, we find now

$$
\begin{array}{r}
1+c+I d-j e+j I f=0 \\
1-c+\frac{1}{I} d-j e-\frac{j}{I} f=0 \\
-1+c+L d+\frac{1}{j} e-\frac{L}{j} f=0 \\
-1-c+\frac{1}{L} d+\frac{1}{j} e+\frac{1}{j L} f=0
\end{array}
$$


The equations corresponding to $(6 \mathrm{a}-6 \mathrm{~d})$ for an incident SV wave are, in our special case, as follows:

$$
\begin{array}{r}
1-d+I c+j f+j I e=0 \\
1+d+\frac{1}{I} c+j f-\frac{j}{I} e=0 \\
-1-d+L c-\frac{1}{j} f-\frac{L}{j} e=0 \\
-1+d+\frac{1}{L} c-\frac{1}{j} f+\frac{1}{j L} e=0
\end{array}
$$

Except for the sign of the transverse waves and the interchange of $c$ with $d$ and of $f$ with $e$, equations (12) and (13) are the same, indicating that the distribution of energy is similar. From equations (13) the following ratios of the square roots of the energies for incident SV waves may be derived:

$$
\begin{aligned}
& \frac{c}{e}=\frac{j I^{\prime}+\frac{1}{j} L^{\prime}}{I^{\prime \prime}-L^{\prime \prime}} \\
& \frac{d}{e}=\frac{1}{2} \frac{j I^{\prime} L^{\prime \prime}+\frac{1}{j} I^{\prime \prime} L^{\prime}}{I^{\prime \prime}-L^{\prime \prime}} \\
& \frac{f}{e}=\frac{1+\frac{1}{j}}{1-\frac{1}{j}} \frac{I L+1}{I-L} \quad I L+1=\frac{1}{2}\left(\cot ^{2} \beta+1\right)
\end{aligned}
$$

where

$$
\begin{array}{ll}
I^{\prime}=I+1 / I ; & L^{\prime}=L+1 / L \\
I^{\prime \prime}=I-1 / I ; & L^{\prime \prime}=L-1 / L
\end{array}
$$

Similar equations hold if a $\mathrm{P}$ wave is incident.

If the square roots of the energy ratios for a wave incident in one medium are $c, d$, $e, f$, and those for a wave incident in the other medium are $C, D, E, F$, for the same angle of incidence, then in our special case the following equations result for an incident SV wave:

$$
\begin{array}{ll}
\frac{c}{C}=-\frac{j^{2} I^{\prime}+L^{\prime}}{j^{2} L^{\prime}+I^{\prime}} ; & \frac{e}{E}=-1 \\
\frac{d}{D}=-\frac{j^{2} I^{\prime} L^{\prime \prime}+I^{\prime \prime} L^{\prime}}{j^{2} I^{\prime \prime} L^{\prime}+I^{\prime} L^{\prime \prime}} ; & \frac{f}{F}=1
\end{array}
$$


and similar equations, with exchanged symbols as stated in connection with equations (13a) to (13d) if a $\mathrm{P}$ wave is incident. For $I^{\prime}, I^{\prime \prime}, L^{\prime}, L^{\prime \prime}$ see $(13 \mathrm{~h})$. Under both conditions, the energies of the refracted waves are the same for waves incident on either side of the discontinuity at the same angle, but in general the distribution of the remaining energy between the two reflected waves differs. For a given angle of incidence, $I$ and $L$ depend on Poisson's ratio only. $j^{2}$ is the ratio of the densities. If $j=1, c=C, d=D$. If $j$ increases, the differences between $c$ and $C$ and also between $d$ and $D$ increase, approaching the ratio of the factors of $j^{2}$ respectively for very large values of $j^{2}$.

If in our special case an SH wave is incident, equation (9) becomes

and equation (8) may be written

$$
H=\frac{\rho_{2}}{\rho_{1}}=\frac{\mu_{2}}{\mu_{1}}
$$

$$
d=\frac{\rho_{1}-\rho_{2}}{\rho_{1}+\rho_{2}}=\frac{\mu_{1}-\mu_{2}}{\mu_{1}+\mu_{2}}=\frac{j^{2}-1}{j^{2}+1} \quad f=\frac{2 \sqrt{\rho_{1} \rho_{2}}}{\rho_{1}+\rho_{2}}=\frac{2 \sqrt{\mu_{1} \mu_{2}}}{\mu_{1}+\mu_{2}}=\frac{2 j}{j^{2}+1}
$$

The energy distribution between the refracted and reflected $\mathrm{SH}$ waves does not depend on the angle of incidence, but only on the ratio of the densities or coefficients of rigidity above and below the discontinuity. $f$ is always positive; $d$ is positive if the wave arrives from the side of greater density, otherwise $d$ is negative.

Results for special cases:

1) $a=0, \beta=0$

$$
\begin{array}{ll}
\text { P wave incident } & d=0, \quad f=0, \quad c=\frac{1-j^{2}}{1+j^{2}}, \quad e=\frac{2 j}{1+j^{2}} \\
\text { SV or SH wave incident } & c=0, \quad e=0, \quad d=\frac{j^{2}-1}{j^{2}+1}, \quad f=\frac{2 j}{1+j^{2}}
\end{array}
$$

While in the SV wave the values of the energy ratios change with the angle of incidence, the values for the $\mathrm{SH}$ waves remain the same as for $\beta=0$.

2) $a=90^{\circ}$

$$
\begin{array}{lll}
\text { P wave incident } & c=-1, & d=e=f=0 \\
\text { SV wave incident } & d=1, & c=e=f=0
\end{array}
$$

3) $\beta=45^{\circ}$. If Poisson's ratio is positive, $\sin a>1$, there can be no incident $\mathrm{P}$ wave. For an $\mathrm{SV}$ wave incident, the energy ratios again are given by (17). Note that in these special cases the energy distribution is the same, except for the sign, whether the incident wave arrives from the side of the greater or the smaller density.

The calculations show that the value of $c$ passes through zero for an incident $P$ wave, and similarly $d$ if an SV wave is incident. For $c=0$, equations (12) lead to

$$
I^{\prime \prime} d+j I^{\prime} f=0 \quad L^{\prime \prime} d-L^{\prime} f / j=0 \quad \text { (using 13h) }
$$

If $f=0, I^{\prime \prime}$ and $L^{\prime \prime}$ must be $0, I=1$ and $L=1$, which requires $a=60^{\circ}, \beta=30^{\circ}$, $q=\sqrt{3}, \sigma=\frac{1}{4}$. Thus, if $\sigma=\frac{1}{4}$, and for that value of Poisson's ratio only, the re- 
flected longitudinal wave, and the refracted transverse wave are simultaneously zero, if a longitudinal wave arrives at an angle of incidence of $60^{\circ}$, regardless of the ratio of the densities. The energy ratios are, in this special case:

$$
c=0, \quad d=\frac{j^{2}-1}{j^{2}+1}, \quad e=\frac{2 j}{j^{2}+1}, \quad f=0
$$

Note the similarity with (16). If an SV wave is incident with $\beta=30^{\circ}$, we have, similarly,

$$
c=\frac{j^{2}-1}{j^{2}+1}, \quad d=0, \quad e=0, \quad f=-\frac{2 j}{j^{2}+1}
$$

In both instances the absolute values are the same, whether the wave arrives in the medium with the smaller or in that with the larger density.

If Poisson's ratio is not $1 / 4$, the system (18) gives a relation between $I$ and $L$, or between $\alpha$ and $\beta$. For a given value of Poisson's ratio, all quantities involved are theoretically determined.

\$II c) Equations referring to the surface of the earth.-If the discontinuity at which the waves arrive is the surface of the earth, $j$ is infinite, and $n, p, r, s, t, e$, and $f$ are zero. In the various systems of four equations the first two are fulfilled, and from the third and fourth we find for $a$

$P$ wave incident

SV wave incident

$$
c=\frac{1-L^{2}}{1+L^{2}} \quad d=\frac{2 L}{L^{2}+1}
$$

SH wave incident

$$
d=-\frac{1-L^{2}}{1+L^{2}} \quad c=\frac{2 L}{L^{2}+1}
$$

$$
d=1
$$

The equations (21) and (22) are similar to several special equations which we have found previously. For comparison with Wiechert (1907) note that his symbol $m$ corresponds to our $L^{2}$.

Results for special cases: For an incident $\mathrm{P}$ wave and $a=0^{\circ}$ or $90^{\circ}, L=$ infinite, $c=-1, d=0$. Similarly, if $\beta=0$ or $90^{\circ}$ in an incident SV wave, $d=1, c=0$. In both instances, the reflected wave of the same type decreases with increasing angle of incidence, reaches a minimum (with a maximum for the reflected wave of the other type), and increases again with the angle of incidence approaching $90^{\circ}$. Jeffreys (1926, p. 326) has pointed out that for relatively large values of Poisson's ratio the amplitudes of the reflected waves of the same type never reach zero, but that they pass twice through zero for Poisson's ratio near 1/4. Generally the value zero is reached for $L=1$. For a given value of Poisson's ratio, equation (4a) furnishes the corresponding value of $q$. Replacing the value of $\sin 2 a$ in equation (7b) for $L$ by $\sin \beta$, using (4b), the condition $L=1$ gives

$$
x^{4}-4 x^{3}+(6-y) x^{2}+(12-y) x+1=0
$$


Removing the root $x=-1$, which has no meaning in our problem,

where

$$
x^{3}-5 x^{2}+(11-y) x+1=0
$$

$$
y=16 / q^{2} \quad x=\cot ^{2} \beta^{*}
$$

$\beta^{*}=$ angle of incidence of SV for which the reflected wave of the same type vanishes. The corresponding $\alpha^{*}$ for $\mathrm{P}$ incident is given by (4b). The maximum value $\sigma^{*}$ of Poisson's ratio for which the reflected wave reaches zero corresponds to an angle $\beta^{*}$ such that $z=\cot ^{2} \beta^{*}$ is a double root of equation (24b). Considering that in (24b) the constant term is $1, z$ must be a solution of

$$
(x-z)^{2}\left(x+\frac{1}{z^{2}}\right)=0
$$

Comparing the coefficients of $x^{2}$ and $x$ in equations (24b) and (26), one finds

$$
\begin{gathered}
2 z^{3}-5 z^{2}-1=0 \\
y=11+\frac{2}{z}-z^{2}
\end{gathered}
$$

The real solution in which we are interested is $z=2.5754$, with $\beta^{*}=31^{\circ} 55^{\prime} 42^{\prime \prime}$, $a^{*}=68^{\circ} 51^{\prime} 24^{\prime \prime}, q=1.76366, \sigma^{*}=0.2631$.

Besides the energy distribution in waves reflected at the surface of the earth, the displacements caused by them are of importance in our problem. In equation (1) the ratio of the total displacement to the arriving wave amplitude is indicated by the letter $Q$. We introduce now the three components of $Q: u$ horizontal in the plane of propagation, $v$ horizontal perpendicular to $u, w$ vertical. To simplify the equations, the ratio of the ground displacement to the incident amplitude is indicated by a single letter (instead of the usual symbols $u / A$, etc.) Using the equations given by Wiechert (1907), we have

$P$ wave incident

$$
u=G \frac{\cos \beta}{\cos 2 \beta} ; \quad v=0 ; \quad w=-\frac{G}{2 \sin \beta}=-G \frac{\cos \beta}{\sin 2 \beta}
$$

where

$$
G=-q \frac{2}{L^{2}+1} \cot 2 \beta
$$

$q=$ ratio of velocities (longitudinal to transverse) as before, $L$ as in (7b).

SV wave incident

$$
u=W \frac{\sin \alpha}{2 \sin ^{2} \beta} ; \quad v=0 ; \quad w=W \frac{\cos \alpha}{\cos 2 \beta}
$$

where

$$
W=\frac{1}{q} \frac{2 L^{2}}{L^{2}+1} \tan 2 \beta
$$

SH wave incident

$$
u=0 ; \quad v=2 ; \quad w=0
$$


In the case of an incident SV wave, $\sin$ a may become greater than 1 ; the equations may then be used with complex quantities. The following equations consider this process (for details see Wiechert, 1907, p. 457; Jeffreys, 1926, p. 328).

$$
\begin{array}{ll}
X=q^{2} \sin ^{2} \beta-1 & Y=q \cos \beta \cot ^{2} 2 \beta \\
Z=1 / \sin \beta \sqrt{Y^{2}+X} & \\
u=Y Z \tan 2 \beta & w=Z \sqrt{X}
\end{array}
$$

Special cases:

1) $a=0, \beta=0$.

2) $\beta=22 \frac{1}{2}{ }^{\circ}$

$$
\begin{array}{lll}
\text { P wave incident } & u=0 & w=2 \\
\text { SV wave incident } & u=2 & w=0
\end{array}
$$

3) $a=90^{\circ}$

$$
\text { P wave incident } \quad u=w
$$

$$
\begin{array}{lll}
\beta=90^{\circ}, & \text { P wave incident } & u=w=0 \\
& \text { SV wave incident } & u=w=0
\end{array}
$$

4) $\alpha=90^{\circ}$,

$$
\text { SV wave incident } u=\frac{2 \cos \beta}{\cos 2 \beta}=\frac{\sqrt{1-\frac{1}{q^{3}}}}{\frac{1}{2}-\frac{1}{q^{2}}} ; \quad w=0
$$

This gives for $u$, if an SV wave is incident at an angle $\beta$ corresponding to $a=90^{\circ}$, the following large values:

$\begin{array}{llllllc}q(\text { velocity ratio }) \ldots \ldots & \sqrt{2} & \sqrt{2.5} & \sqrt{3} & \sqrt{4} & \sqrt{5} & \infty \\ \text { Poisson's ratio....... } 0.000 & 0.167 & 0.250 & 0.333 & 0.375 & 0.500 \\ u \ldots \ldots \ldots \ldots \ldots \ldots \ldots & \infty & 9.165 & 4.842 & 3.464 & 2.981 & 2.000\end{array}$

5) $\beta=45^{\circ}$.

$$
\text { SV wave incident } \quad u=0 ; \quad w=\sqrt{2}
$$

\section{§ III. Calculated Values}

$\S$ III a) General cases.-There are three groups of discontinuities in the earth for which the knowledge of the energy distribution is required for the study of wave amplitudes. The first contains the boundaries between the layers of the earth's crust; the second, the hypothetical transitions from crystalline to amorphous material at a temperature above the melting point; and the third, the boundary of the core and possible discontinuities inside the core involving at least one layer with very small rigidity so that transverse waves in such a layer may be neglected. We will not consider this last group here. The study of shocks in California indicates that the ground displacements in the transverse waves are usually between five and ten times those in the longitudinal waves. Consequently, a reflection from an $\mathbf{S}$ wave 
produced by an earthquake may lead to transformed $\mathrm{P}$ waves with amplitudes of the same order of magnitude as those in the direct $P$ waves if the square root of the ratio of the energy in a transformed wave (SV into $\mathrm{P}$ ) to that in the incident SV wave is only 0.1 . Thus, such transformed waves cannot be neglected, and studies in which these waves were not considered are likely to lead to incorrect conclusions in our problem. In artificial explosions the energy of the $\mathrm{S}$ waves is relatively smaller, and a much larger fraction of an original $S$ wave must be transformed into a longitudinal wave to produce a similar effect. However, it must be considered that in seismic prospecting the direct $\mathrm{P}$ waves are usually recorded only with a small fraction of the sensitivity used in the recording of the reflected waves, and waves transformed from $P$ into $S$ may be large enough under favorable circumstances to produce phases with amplitudes of the same order of magnitude as those of late longitudinal waves.

TABLE 1

Sotrces for Points Used in Figures 1 and 2

\begin{tabular}{|c|c|c|c|c|}
\hline \multirow{2}{*}{ Curve } & \multirow{2}{*}{$P$ ineident } & \multicolumn{2}{|c|}{ SV incident from } & \multirow{2}{*}{ SH incident } \\
\hline & & Above & Below & \\
\hline 1 & Slichter-Gabriel case 1 & Nordquist, Richter & Slichter-Gabriel & Nordquist \\
\hline 2 & Blut & Fu, Silgado & Ergin & Nordquist \\
\hline 3 & Knott & Knott & Knott & Gutenberg \\
\hline 4 & Slichter-Gabriel case 2 & \multicolumn{2}{|c|}{ Slichter-Gabriel case 2} & Nordquist \\
\hline
\end{tabular}

The investigations which contain data useful for our purpose are those of Knott (1899), Jeffreys (1926), Blut (1932), Slichter and Gabriel (1938), and Muskat and Meres (1940). Except for $\mathrm{SH}$, the last-named contains data for small angles of incidence only, not exceeding $30^{\circ}$ for incident $\mathrm{P}$ waves and $17^{\circ}$ for $\mathrm{SV}$ waves. The other authors considered only $\mathrm{P}$ or SV waves. Points of the missing curves were calculated by Dr. C. F. Richter and Mr. J. M. Nordquist of the Seismological Laboratory and by the author (all to 5 places), and by Messrs. K. Ergin, C. Y. Fu, and E. Silgado, graduate students in Geophysics at the California Institute of Technology, by use of a slide rule. ${ }^{3}$ As the calculations are tedious, only a limited number of points has been calculated for the new curves (table 2 ) as well as those available in the literature. Consequently, the curves reproduced in figures 1 and 2 may be inaccurate in details, especially figure $2, c$ and $g$. One or the other irregularity plotted in the figures may be due to errors in calculations ${ }^{4}$ or rounding off, and others may be missing entirely. Equation (4c) offers a check on the results, but usually one or more of the figures involved are so small that errors affect only the third or fourth decimal of the sum of the squares. In general it is unlikely that the deviations of the curves from the correct values are significant for their use in the calculation of amplitudes.

3 The author is very grateful to those mentioned who have participated in the calculations, to Mr. H. O. Wood (as in many previous instances) and to Dr. C. F. Richter for suggestions in revising the manuscript, and to Mr. John M. Nordquist for drafting the figures.

4 Several misprints and errors in the values given by Knott have been corrected, but others are suspected. The somewhat unusual assumptions made by Knott did not justify the recalculation of all values. 
Four sets of assumptions are used to plot the square roots of the energy ratios in figures 1 (reflected and transmitted waves of the same type) and 2 (waves with change from $\mathrm{P}$ to $\mathrm{SV}$ and vice versa). The sources for the points used may be found in table 1. In addition, a few points were calculated by the author, and the results for special cases discussed in the section on theory were used. Figure 2 leaves no doubt that in earthquakes (but very rarely in artificial explosions) waves transformed from SV into $P$ under favorable conditions may have about the same amplitudes as the direct $P$ waves. The refracted waves of the same kind as the incident
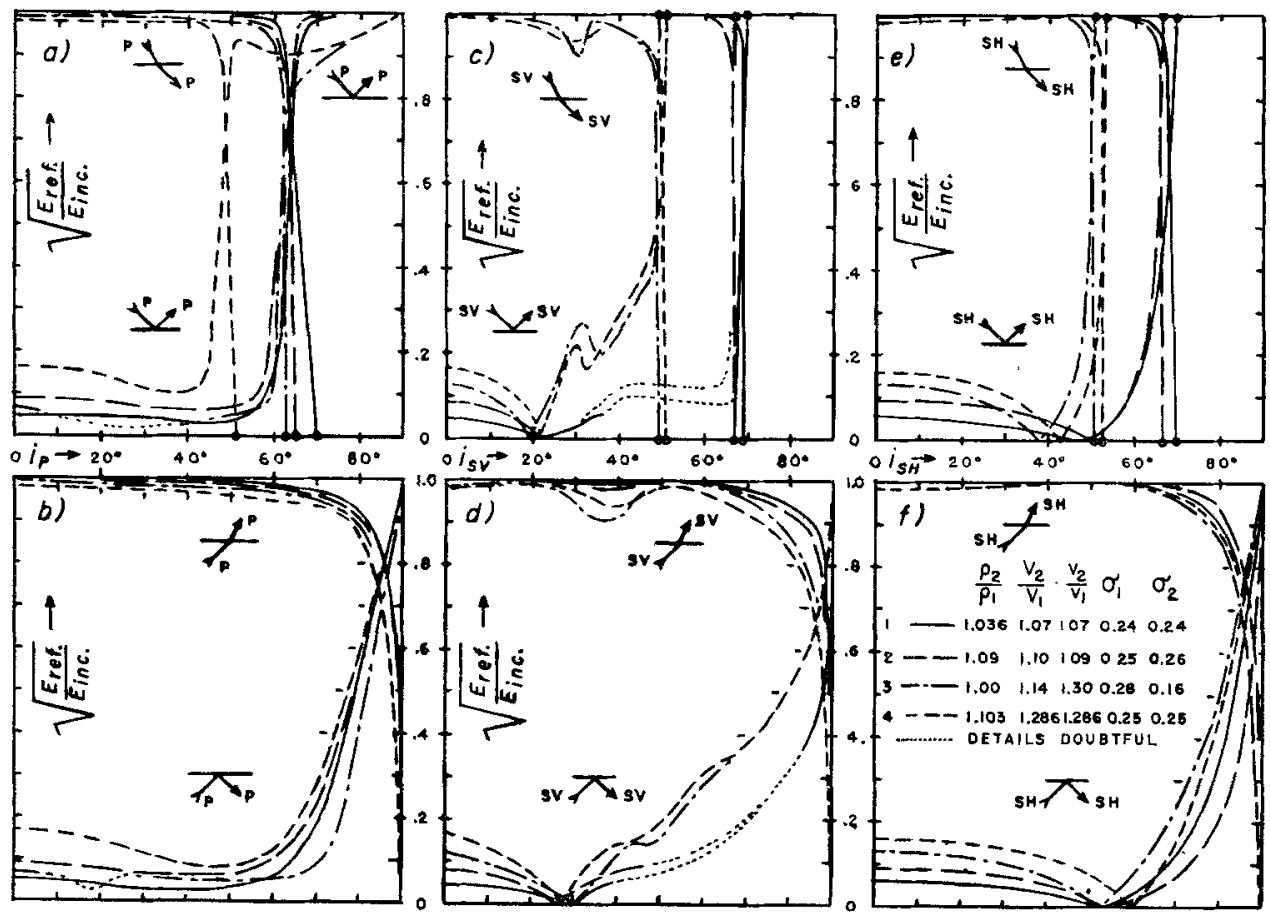

Fig. 1. Square roots of ratio reflected or transmitted to incident energy as function of angle of incidence, $i$, if no change in wave type occurs. Subscript " 1 " refers to upper, subscript "2" to lower, medium. Other symbols are indicated in the figure.

waves do not lose an appreciable amount of energy in passing discontinuities except for certain conditions such as near-grazing incidence of the refracted waves. It is intended to discuss in a future paper applications of figures 1 to 3 and of equation (1) to the crustal layers.

$\$$ III b). Distribution of energy for waves arriving at a discontinuity with no change in velocities.-Calculations for the conditions which have been treated theoretically in section II $b$, have been carried out by Jeffreys (1926, p. 332) on the assumption that the ratio of the densities is 0.4 . His results are of interest mainly for a study of the behavior of the energy ratios as a function of the angle of incidence. Values for density ratios $0.7,0.8,0.9,1.1,1.2$, and 1.3 have been given by Muskat and Meres $(1940$, columns $\bar{a}=1.00$ ), but only for the small angles of incidence mentioned in section III a. 
From the theory, it follows that except for the signs the distribution of energy is the same for incident $\mathbf{P}$ and SV waves if one compares refracted waves of the same kind, refracted transformed waves, etc.; from equations (14) the results may be found for waves incident from one side if they are known for incidence from the
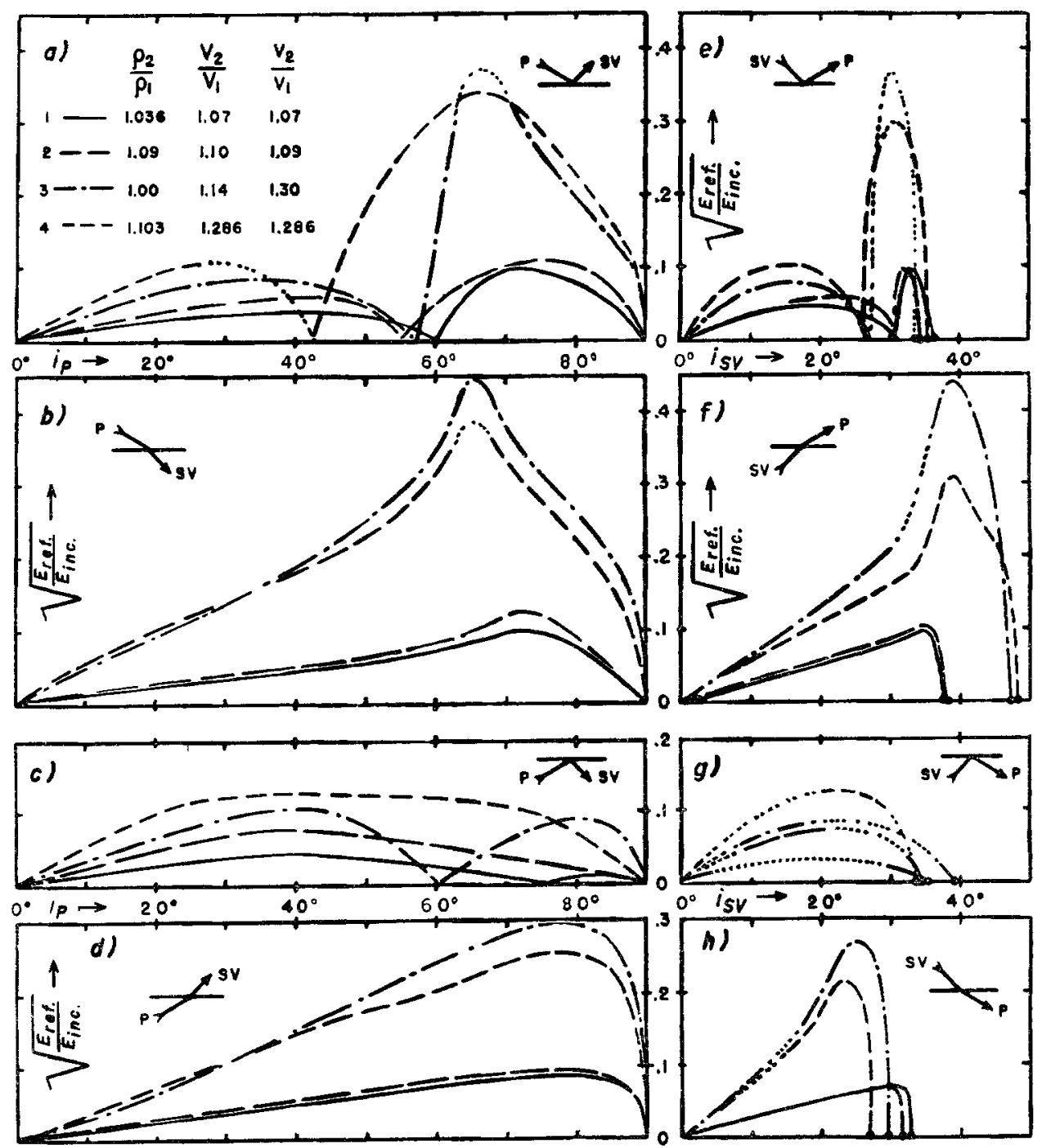

Fig. 2. Square root of ratio reflected or transmitted to incident energy as function of angle of incidence $i$, if incident and reflected or transmitted wave are of different type. Symbols as in figure 1 .

other. Consequently, detailed calculations have to be made for one kind of incident waves and for one direction (from smaller to greater density or vice versa) only.

Table 3 contains the results calculated by the author to 5 places for incident SV waves for transition from a layer with higher density to one with lower (ratio 1.1 to 1 ) and assuming Poisson's ratio $\sigma=1 / 4$. The figures have not been plotted since 
TABLE 2

Calculated Values of Square Roots of Energy Ratios

(For explanation of symbols see section II a)

a) SV incident from above, curve 1; for assumptions see figures 1 and 2 .

\begin{tabular}{|c|c|c|c|c|c|c|c|c|c|c|c|}
\hline & $\beta$ & & $a$ & & 5 & & $\eta$ & $d$ & $c$ & $f$ & $e$ \\
\hline $0^{\circ}$ & $00^{\prime}$ & $0^{\circ}$ & $00^{\prime}$ & $0^{\circ}$ & $00^{\prime}$ & $0^{\circ}$ & $00^{\prime}$ & $\begin{array}{ll}-0 & 02\end{array}$ & 0.000 & -09997 & 0.00 \\
\hline 20 & 00 & 35 & 55.5 & 21 & 28.3 & 38 & 523 & -0.01 & -0.045 & -0.99 & 0.05 \\
\hline 29 & 00 & 56 & 16.7 & 31 & 15.5 & 62 & 49 & +0.02 & -0.017 & -0997 & 0.07 \\
\hline 30 & 00 & 59 & 04,2 & 32 & 21.3 & 66 & 33.2 & 0.03 & -0.010 & -099 & 0.07 \\
\hline 31 & 00 & 62 & 04.8 & 33 & 272 & 70 & 548 & 0.03 & +0.002 & -0996 & 0.08 \\
\hline 32 & 00 & 65 & 231 & 34 & 333 & 76 & 29.4 & 004 & 002 & -0996 & 0.08 \\
\hline 33 & 01.5 & 69 & 13.7 & 35 & 41 & 90 & 00.0 & +0.05 & 0.10 & -099 & 0.00 \\
\hline 34 & 00 & 73 & 27.6 & 36 & 45.9 & & $\ldots$ & +0.05 & 0.11 & -0.99 & $\ldots$ \\
\hline 35 & 39.2 & 90 & 00 & 38 & 36 & & . & +005 & 0.00 & -0998 & $\ldots$ \\
\hline 37 & 00 & & $\ldots$ & 40 & 06 & & $\ldots$ & 0.06 & $\ldots$ & -0.998 & $\ldots$. \\
\hline 45 & 00 & & $\ldots$ & 49 & 11 & & $\ldots$ & 0.09 & $\ldots$ & -0.99 & $\ldots$ \\
\hline 60 & 00 & & $\cdots$ & 67 & 57.8 & & $\cdots$ & $\begin{array}{ll}0 & 09\end{array}$ & & -0.99 & $\ldots$ \\
\hline 65 & 00 & & $\cdots$ & 75 & 52.3 & & $\ldots$ & 008 & $\ldots$ & -0.99 & $\ldots$ \\
\hline 69 & 06.8 & & $\ldots$ & 90 & 00 & & $\ldots$ & 100 & $\ldots$ & 0.00 & $\ldots$ \\
\hline
\end{tabular}

b) SV incident from above, curve 2; for assumptions see figure 1 .

\begin{tabular}{|c|c|c|c|c|c|c|c|c|}
\hline $0^{\circ}$ & $00^{\prime}$ & $0^{\circ} \quad 00^{\prime}$ & $0^{\circ} \quad 00^{\prime}$ & $0^{\circ} \quad 00^{\prime}$ & -009 & 0.00 & -0.99 & 0.00 \\
\hline 20 & 00 & $\begin{array}{ll}36 & 18\end{array}$ & $21 \quad 34$ & $40 \quad 32$ & -0.04 & -0.06 & -0.99 & 0.04 \\
\hline 29 & 00 & $56 \quad 56$ & $31 \quad 28$ & $\begin{array}{ll}67 & 13\end{array}$ & +0.02 & -0.04 & -0.99 & 0.07 \\
\hline 30 & 30 & $61 \quad 21$ & $\begin{array}{ll}33 & 03\end{array}$ & $74 \quad 51$ & 0.03 & -0.02 & -0.99 & 0.08 \\
\hline 31 & 45 & 6530 & $34 \quad 57$ & $90 \quad 00$ & 0.07 & +0.08 & -099 & 0.00 \\
\hline 34 & 42 & $90 \quad 00$ & $\begin{array}{ll}38 & 18\end{array}$ & . & 0.07 & 0.00 & -099 & . \\
\hline 45 & 00 & $\ldots$ & $49 \quad 28$ & & 012 & . . & -0.99 & $\ldots$ \\
\hline 60 & 00 & $\ldots$ & $68 \quad 45$ & & 0.12 & & -0.99 & $\cdots$ \\
\hline 66 & 46 & $\ldots$ & $90 \quad 00$ & & 100 & $\ldots$ & 000 & $\ldots$ \\
\hline
\end{tabular}

c) SV incident from below, curve 2; same assumptions as for b).

\begin{tabular}{r|r|r|r|r|r|r|r}
\hline 0.0 & 0.0 & 0.0 & 0.0 & +0.08 & 0.00 & -0.99 & 0.00 \\
24.2 & 450 & 22.5 & 405 & +0.01 & 0.06 & -0.99 & -0.07 \\
34.7 & 90.0 & 313 & 65.5 & -0.07 & 0.00 & -0.99 & -0.10 \\
38.8 & $\ldots$ & 352 & 90.0 & -007 & $\ldots$ & -0.99 & -0.00 \\
450 & $\ldots$. & 405 & $\ldots$. & -0.11 & $\ldots$ & -0.99 & $\ldots$ \\
65.0 & $\ldots$ & 570 & $\ldots$. & -016 & $\ldots$ & -0.99 & $\ldots$ \\
85.0 & $\ldots$ & 670 & $\ldots$. & -041 & $\ldots$. & -092 & $\ldots$ \\
900 & $\ldots$ & 66.8 & $\ldots$ & -100 & $\ldots$ & 0 & 00 \\
\hline
\end{tabular}




\section{TABLE 2-Continued}

d) SH incident. For incidence from above, $\beta$ is the angle of incidence; for incidence from below, $\zeta$ is to be used and the signs of $d$ are to be changed. All values of $f$ are negative. For assumptions, see figure 1.

\begin{tabular}{|c|c|c|c|c|c|c|c|c|c|c|c|c|c|}
\hline \multirow{2}{*}{$1,2,4$} & \multicolumn{3}{|c|}{ Curve 1} & \multicolumn{3}{|c|}{ Curve 2} & \multicolumn{3}{|c|}{ Curve 4} & \multicolumn{4}{|c|}{ Curve 3} \\
\hline & $\beta$ & $d$ & $f$ & $\beta$ & $d$ & $f$ & $\beta$ & $d$ & $f$ & $\zeta$ & $\beta$ & $d$ & $f$ \\
\hline $0: 0$ & 0.0 & $-0 \quad 06$ & 100 & $0: 0$ & -0.09 & 099 & 0.0 & -0.16 & 0.98 & $0^{\circ}$ & $0: 0$ & -0.13 & 0.99 \\
\hline 113 & 103 & -005 & 100 & 10.4 & -0.09 & 0.99 & 90 & $-\begin{array}{ll}-0 & 16\end{array}$ & 0.99 & 10 & 7.7 & -0.13 & 0.99 \\
\hline 26.6 & 251 & -004 & 1.00 & 242 & -008 & 099 & 208 & -0.14 & 0.99 & 20 & 15.3 & -0.12 & 0.99 \\
\hline 33.7 & 315 & $-\begin{array}{ll}-0 & 03\end{array}$ & 100 & 30.6 & -0.07 & 100 & 26.2 & -0.13 & 099 & 30 & 22.6 & -010 & 0.99 \\
\hline 450 & 41.3 & $-\begin{array}{ll}-0 & 02\end{array}$ & 1.00 & 40.5 & -0.05 & 1.00 & 342 & -0.09 & 1.00 & 40 & 296 & -0.07 & 1.00 \\
\hline 531 & 48.2 & 000 & 100 & 472 & -002 & 1.00 & 39.6 & -0.06 & 1.00 & 50 & 364 & -0.02 & 1.00 \\
\hline 63.4 & 563 & 005 & 1.00 & 55.1 & 0.04 & 1.00 & 45.3 & 0.06 & 100 & 60 & 417 & 007 & 1.00 \\
\hline 760 & 643 & 035 & 094 & 63.0 & 0.22 & 098 & 505 & 0.30 & 0.95 & 70 & 463 & 0.22 & 0.98 \\
\hline 84.3 & 685 & 054 & 0.84 & 65.9 & 041 & 0.91 & 52.4 & 0.63 & 0.78 & 80 & 492 & 0.49 & 0.87 \\
\hline 85.7 & 689 & 063 & 078 & 66.3 & 064 & 077 & 52.5 & 0.71 & 0.71 & 85 & 500 & 070 & 0.71 \\
\hline 87.1 & 690 & 0.73 & 0.68 & 664 & 0.74 & 0.67 & 527 & 079 & 0.61 & 88 & 502 & 087 & 0.50 \\
\hline 90.0 & 693 & 1.00 & 000 & 666 & 1.00 & 000 & 52.8 & 100 & 0.00 & 90 & 503 & 100 & 0.00 \\
\hline
\end{tabular}

TABLE 3

SQdare Roots of Energy Ratios For Incident SV Wave, if $V_{1}=V_{2}, v_{1}=v_{2}, \rho_{1} / \rho_{2}=1.1, \sigma=1 / 4$ ( $\beta=$ angle of incidence of SV; $\alpha=$ angle of incidence of $\mathrm{P}$ waves.)

\begin{tabular}{|c|c|c|c|c|c|c|c|c|c|}
\hline & & & & & & \multicolumn{4}{|c|}{ Square root of energy ratio (to incident energy) for } \\
\hline \multicolumn{3}{|c|}{$\beta$} & \multicolumn{3}{|c|}{$a$} & P refl. & S refl. & P transm. & $\mathrm{S}$ transm. \\
\hline $0^{\circ}$ & $0^{\prime}$ & $0^{\prime \prime}$ & $0^{\circ}$ & $0^{\prime}$ & $0^{\prime \prime}$ & 0000 & +0.048 & 0000 & -0.999 \\
\hline 5 & 45 & 14 & 10 & 0 & 0 & +0 014 & +0.046 & -0001 & -0999 \\
\hline 11 & 23 & 20 & 20 & 0 & 0 & +0026 & +0.040 & -0.003 & -0.999 \\
\hline 16 & 46 & 45 & 30 & 0 & 0 & +0036 & +0.032 & -0.001 & -0999 \\
\hline 30 & 0 & 0 & 60 & 0 & 0 & +0.048 & 0000 & 0.000 & -0999 \\
\hline 34 & 0 & 0 & 75 & 355 & & +0.047 & -0011 & +0011 & -0.999 \\
\hline 35 & 0 & 0 & 83 & 27.0 & & +0.053 & -0014 & +0028 & -0.998 \\
\hline 35 & 15 & 0 & 88 & 29.4 & & +0.089 & -0.009 & +0076 & -0.993 \\
\hline 35 & 15 & 47 & 89 & 30 & 0 & +0.146 & +0.005 & +0.137 & -0.980 \\
\hline 35 & 15 & 51 & 89 & 50 & 0 & +0.239 & +0.042 & +0231 & -0.942 \\
\hline 35 & 15 & 512 & 89 & 59 & 0 & +0.494 & +0370 & +0485 & -0620 \\
\hline 35 & 15 & 518 & 89 & 59 & 59 & +0163 & +0973 & +0.160 & -0.027 \\
\hline 35 & 15 & 518 & 90 & 0 & 0 & +0000 & +1.000 & 0.000 & 0000 \\
\hline 35 & 30 & 0 & . & $\ldots$ & & . . . & -0.017 & $\ldots \ldots$ & -09999 \\
\hline 45 & 0 & 0 & & $\ldots$ & & $\ldots$ & -0.048 & $\ldots$ & -0999 \\
\hline 89 & 0 & 0 & & $\ldots$ & & $\ldots$ & -0.047 &.$\ldots$ & -0.999 \\
\hline 90 & 0 & 0 & . & . & & . . & +1.000 & & 0000 \\
\hline
\end{tabular}


the main feature, the large changes when a approaches $90^{\circ}$, are connected with a very small variation of $\beta$. From the data it is clear that similar rapid changes in the values occur after the passage of the critical angle, and also when $\beta$ approaches $90^{\circ}$.

The angle of incidence for which the rapid changes occur depends only on Poisson's ratio, which is necessarily the same in both layers since the velocities of longi-
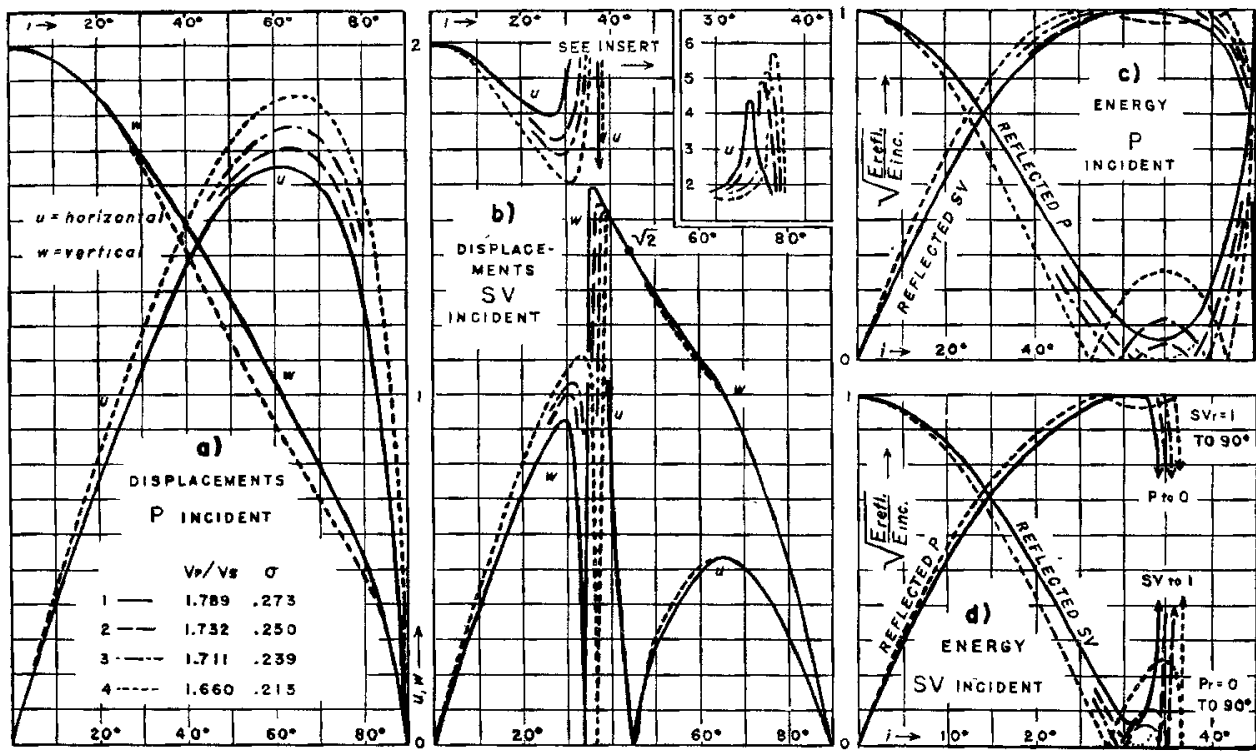

Fig. 3. Ratio of horizontal $(u)$ and vertical $(w)$ ground displacements to incident amplitude at the surface of the earth, and square roots of ratio reflected to incident energy as function of angle of incidence $i$ for various velocity ratios $V_{P}$ (longitudinal) to $V_{S}$ (transverse) and corresponding Poisson's ratio $\sigma$. Note that scales in insert, $b$, differ from those in the main figure.

TABLE 4

Square Roots (Absoldte Values) of the Ratio of Energy $d$ in Reflected and $f$ in Trans-

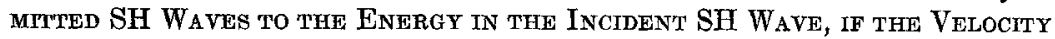
of Transverse Waves is the Same on Both Sides of the Discontinutty

\begin{tabular}{|c|c|c|c|c|c|c|c|c|c|c|}
\hline Density ratio . . . . $\quad \ldots$ & 1.00 & $\begin{array}{l}1.05 \\
0.952\end{array}$ & $\begin{array}{l}1.10 \\
0.909\end{array}$ & $\begin{array}{l}1.15 \\
0.870\end{array}$ & $\begin{array}{l}1.20 \\
0.833\end{array}$ & $\begin{array}{l}1.3 \\
0.769\end{array}$ & $\begin{array}{l}1.5 \\
0.667\end{array}$ & $\begin{array}{l}2.0 \\
0.5\end{array}$ & $\begin{array}{l}5.0 \\
0.2\end{array}$ & $\begin{array}{c}\infty \\
0.00\end{array}$ \\
\hline & 0.0 & & 0.048 & 0.070 & 0091 & 0130 & 0200 & 0333 & 0667 & 1.00 \\
\hline Transmitted $(f)$. & 1000 & 09997 & 0.999 & 0.998 & 0996 & 0991 & 0.980 & 0943 & 0745 & 0 \\
\hline
\end{tabular}

tudinal and transverse waves are respectively the same. If the velocities on both sides of the discontinuity differ, the curves for incident SV waves still show peculiarities of a similar kind (fig. 1, $c$ and $d$ ) near the critical angle, which seem to exist also if there is no difference in density in the two layers (curves 3 ). In our special case (equal velocities on both sides) the changes in energy ratio near the critical angle occur the more rapidly with changing angle of incidence the smaller the difference between the two densities.

If an SH wave is incident, the distribution of energy depends only on the ratio of the densities, not on the angle of incidence. Table 4 contains a few characteristic 
TABLE 5

Calculated Square Roots $c$ and $d$ of the Energy Ratios of the Reflected to the Incident Energy, and Relative Ground Displacements $u$ (Horizontal) and $w$ (Vertical) at the Surface of the Earth

(Symbols as in section II a. For assumptions, see figure 3.)

a) Additional values for curves 1 , figure $3, b$.

\begin{tabular}{c|cccccccccc}
\hline \hline$\beta$ & $35^{\circ}$ & $36^{\circ}$ & $37^{\circ}$ & $38^{\circ}$ & $40^{\circ}$ & $45^{\circ}$ & $50^{\circ}$ & $63: 4$ & $70^{\circ}$ & $80^{\circ}$ \\
\hline$u$ & 3.14 & 2.23 & 164 & 1.22 & 0.66 & 000 & 028 & 0.53 & 0.49 & 031 \\
$w$ & 131 & 154 & 160 & 1.60 & 1.55 & 1.41 & 1.29 & 1.09 & 0.90 & 0.52 \\
\hline
\end{tabular}

b) Additional values for curves 2 , figure $3, b$.

\begin{tabular}{c|ccccccc}
\hline$\beta$ & $37^{\circ}$ & $38^{\circ}$ & $39^{\circ}$ & $40^{\circ}$ & $42^{\circ}$ & $45^{\circ}$ & $50^{\circ}$ \\
\hline$u$ & 2.09 & 145 & 1.04 & 074 & 035 & 0.00 & 0.29 \\
$w$ & 1.55 & 1.58 & 1.57 & 155 & 1.49 & 1.41 & 1.30 \\
\hline
\end{tabular}

c) Values for curves 3, figure 3 . For incident $P$ waves $a$ is angle of incidence, for incident SV waves $\beta$ is to be used, and the values of $c$ and $d$ are to be exchanged.

\begin{tabular}{|c|c|c|c|c|c|c|c|c|c|c|c|}
\hline \multirow{2}{*}{$a$} & \multirow{2}{*}{\multicolumn{2}{|c|}{$\beta$}} & \multirow{2}{*}{. } & \multirow{2}{*}{$d$} & \multicolumn{2}{|c|}{$P$ incident } & \multicolumn{2}{|c|}{ SV incident } & \multirow{2}{*}{$\beta$} & \multicolumn{2}{|c|}{ SV incident } \\
\hline & & & & & $u$ & $w$ & $u$ & $w$ & & $u$ & $w$ \\
\hline $5^{\circ}$ & $2^{\circ}$ & $55: 3$ & -0988 & 0155 & 020 & 199 & 2.00 & 012 & $37^{\circ}$ & 2.42 & 1.52 \\
\hline 10 & 5 & 49.6 & -0.952 & 0306 & 041 & 197 & 198 & 0.24 & 38 & 1.60 & 1.57 \\
\hline 20 & 11 & 31.9 & -0816 & $\begin{array}{l}0579 \\
\end{array}$ & 079 & 185 & 193 & 0.46 & 39 & 1.11 & 1.57 \\
\hline 30 & 16 & 597 & -0612 & 0791 & 1.14 & 168 & 186 & 0.66 & 40 & 0.78 & 1.55 \\
\hline 40 & 22 & 042 & -0.375 & 0916 & 1.41 & 1.45 & 1.75 & 0.82 & 43 & 0.21 & 1.46 \\
\hline 50 & 26 & $\begin{array}{ll}361 & 1\end{array}$ & -0.142 & 0989 & 1.64 & 122 & 1.69 & 096 & 45 & 000 & 141 \\
\hline 60 & 30 & 24.8 & 0.042 & 0 999 & 1.76 & 098 & 1.70 & 1.03 & 50 & 030 & 130 \\
\hline 65 & 31 & 59.4 & 0.100 & $\begin{array}{l}0995 \\
\end{array}$ & 1.77 & 0.86 & 1.73 & 1.03 & 60 & 0.51 & 112 \\
\hline 70 & 33 & 191 & 0124 & 0992 & 175 & 075 & 1.85 & 1.02 & 65 & 0.52 & 102 \\
\hline 75 & 34 & 225 & 0.096 & 0995 & 166 & 0.65 & 2.06 & 0.97 & 70 & 0.50 & 0.90 \\
\hline 80 & 35 & 088 & -0016 & $\begin{array}{ll}0999\end{array}$ & 1.46 & 0.52 & 2.46 & 0.85 & 80 & 031 & 0.52 \\
\hline 85 & 35 & 368 & -0297 & 0955 & 1.03 & 0.35 & 3.28 & 0.59 & & & \\
\hline 90 & 35 & 463 & -1.000 & 0.000 & 000 & 0.00 & 5.13 & 0.00 & & & \\
\hline
\end{tabular}

d) Values for curves 4, figure 3. Details as in c).

\begin{tabular}{|c|c|c|c|c|c|c|c|c|c|c|}
\hline \multirow{2}{*}{$a$} & \multirow{2}{*}{$\beta$} & \multirow{2}{*}{$c$} & \multirow{2}{*}{$d$} & \multicolumn{2}{|c|}{$P$ incident } & \multicolumn{2}{|c|}{ SV incident } & \multirow{2}{*}{$\beta$} & \multicolumn{2}{|c|}{ SV incident } \\
\hline & & & & $u$ & $w$ & $u$ & $w$ & & $u$ & $w$ \\
\hline $40^{\circ}$ & $22^{\circ} 47^{\prime} \cdot 1$ & -0.315 & 0949 & 1.47 & 144 & 1.73 & 088 & $39^{\circ}$ & 1.42 & 1.56 \\
\hline 50 & $\begin{array}{lll}27 & 29 & 2\end{array}$ & $\begin{array}{lll}-0 & 059\end{array}$ & 0998 & 169 & 1.19 & 164 & 102 & 40 & 0.93 & 155 \\
\hline 60 & $\begin{array}{lll}31 & 27 & 1\end{array}$ & 0.119 & 0.989 & 1.83 & 0.93 & 159 & 110 & 41 & 0.64 & 152 \\
\hline 70 & 34309 & 0254 & 0967 & 185 & 0.71 & 171 & 1.11 & 42 & 0.40 & 1.49 \\
\hline 80 & $\begin{array}{lll}36 & 23 & 7\end{array}$ & 0151 & 0.989 & 161 & 0.50 & 231 & 0.97 & 50 & 031 & 1.30 \\
\hline 85 & $\begin{array}{lll}36 & 53 & 1\end{array}$ & -0127 & 0992 & 121 & 0.35 & 323 & 073 & 60 & 0.52 & 112 \\
\hline \multirow[t]{2}{*}{90} & $\begin{array}{llll}37 & 03 & 0\end{array}$ & -1000 & 0000 & 0.00 & 0.00 & 583 & 0.00 & 70 & 050 & 0.88 \\
\hline & $>37030$ & -1000 & 0000 & . . & & & $\rightarrow$ & 80 & 0.31 & 051 \\
\hline
\end{tabular}


values. For an angle of incidence of $0^{\circ}$ these are the same as for SV waves, and, together with the special results in section II b and table 3, may be used to draw curves giving a first approximation of the energy distribution in all instances.

\$III c) Results referring to reflection at the surface of the earth and to the ratio of ground amplitudes to the amplitudes of the arriving waves.-A few data on the energy distribution for waves reflected at the surface of the earth were given by Knott (1899). Other sets of data and corresponding values for the ratio of ground amplitudes to the amplitudes of the incident waves were calculated by Gutenberg (Zoeppritz, Geiger, and Gutenberg, 1912; Geiger and Gutenberg, 1912) and by Jeffreys (1926, p. 327).

In figure 3, curves corresponding to four values of Poisson's ratio are reproduced. The data for curve 1 were taken from the older calculations of the author, those for curve 2 from Jeffreys (1926); for both, a few additional points were calculated (table 5, a and b), especially where large changes are indicated in figure $3, b$. All points on which curves 3 and 4 are based were newly calculated to 5 places by the author (table 5, $c$ and $d$ ). In addition, the special results mentioned in section II c were used. Most of the important features have been discussed by Jeffreys. The peculiar behavior of $w$ near the critical angle in figure $3, b$, seems to have escaped attention hitherto.

For an incident SH wave, all the energy is reflected into an SH wave, the horizontal ground displacement is always twice the amplitude of the incident wave, and the vertical displacement always zero regardless of the angle of incidence or Poisson's ratio.

\title{
§ IV. Summary
}

The investigation of amplitudes of elastic waves passing through layers of the earth, either in earthquakes or from artificial explosions, requires a knowledge of the distribution of energy at points of reflection or refraction, and also of the ratio between the amplitudes arriving at the surface of the earth and the displacements of the ground there. Some fundamental theoretical problems involved are discussed (section II) and typical curves for the quantities mentioned are given (section III).

\author{
California Institute of Technology \\ Pasadena, Califoria \\ (Batch Gradtate School of the Geological Sctences, contributron no. 359)
}


ButT, H.

\section{REFERENCES}

1932. "Ein Beitrag zur Theorie der Reflexion und Brechung elastischer Wellen an Unstetigkeitsflächen," Zeitschr.f. Geophysik, 8:130-114, 305-322.

BYeRLY, P.

1942. "Elastic Waves," Seismology, pp. 152-178.

Dix, C. H.

1939. "Refraction and Reflection of Seismic Waves," Geophysics, 4:81-101.

Getger, L., and B. Gutenberg

1912. "Ueber Erdbebenwellen, VI," Nachr. Gesell. d. Wiss. Gättingen, math.-phys. Kl., pp. $623-675$.

GUTENBERG, B.

1932. "Theorie der Erdbebenwellen," Handbuch der Geophysik, 4:42-57.

JEFFREYS, $\mathrm{H}$.

1926. "The Reflexion and Refraction of Elastic Waves," Mon. Not. Roy. Astron. Soc., Geophys. Suppl., 1:321-334.

Joos, G., and J. TeLrow

1939. "Zur Deutung der Knallwellenausbreitung an der Trennschicht zweier Medien," Physikal. Zeitschr., 40:289-293.

KNotт, C. G.

1899. "Reflexion and Refraction of Elastic Waves with Seismological Applications," Phrl. $M a g ., 5$ th ser., 48:64-97.

Macelwane, J. B.

1933. "Reflection and Refraction of Earthquake Waves," Physics of the Earth, VI, Bull. Nat. Res. Council, no. 90: 116-120.

1936. "Reflection and Refraction of Seismic Waves," Introduction to Theoretical Seismology, pp. 147-179.

MUSKAT, M.

1938. "The Reflection of Longitudinal Wave Pulses from Plane Parallel Plates," Geophyszcs, 3:198-218.

Muskat, M., and M. W. Meres

1940. "Reflection and Transmission Coefficients for Plane Waves in Elastic Media," Geophysics, 5:115-148.

OтT, H.

1942. "Reflexion und Brechung von Kugelwellen," Annalen, d. Physik (5) Vol. 41, pp. 443-466.

Slichter, L. B., and G. Gabriel

1933. "Studies in Reflected Seismic Waves," Gerlands Beitr. z. Geophysı, 38:228-238.

WIECHERT, E.

1907. "Ueber Erdbebenwellen, I," Nachr. Gesell. d. Wiss. Göttingen, math.-phys. Kl., pp. $415-589$.

Zoeprritz, K., L. Getger, and B. Gutenberg

1912. "Ueber Erdbebenwellen, V," Nachr. Gesell. d. Wiss. Göttingen, math.-phys. Kl., pp. $121-206$. 